\title{
Effect of Nitric Oxide on Paraquat-Tolerance in Lettuce Leaves
}

\author{
Jee-Na Lee, Jung-Hee Hong ${ }^{*}$ \\ Department of Biological Sciences, Pusan National University, Busan 609-735, Korea
}

(Manuscript received 10 September, 2011; revised 23 November, 2011; accepted 10 December, 2011)

\begin{abstract}
The protective effect of nitric oxide (NO) on the antioxidant system under paraquat(PQ) stress was investigated in leaves of 8-week-old lettuce (Lactuca sativa L.) plants. PQ stress caused a decrease of leaf growth including leaf length, width and weight. Application of NO donor, sodium nitroprusside (SNP), significantly alleviated PQ stress induced growth suppression. SNP permitted the survival of more green leaf tissue preventing chlorophyll content reduction and of higher quantum yield for photosystem $\Pi$ than in non-treated controls under PQ exposure, suggesting that NO has protective effect on chloroplast membrane in lettuce leaves. Flavonoids and anthocyanin were significantly accumulated in the leaves upon PQ exposure. However, the rapid increase of these compounds was alleviated in the SNP treated leaves. PQ treatment resulted in lipid peroxidation and induced accumulation of hydrogen peroxide $\left(\mathrm{H}_{2} \mathrm{O}_{2}\right)$ in the leaves, while SNP prevented PQ induced increase in malondialdehyde (MDA) and $\mathrm{H}_{2} \mathrm{O}_{2}$. These results demonstrate that $\mathrm{SNP}$ serves as an antioxidant agent able to scavenge $\mathrm{H}_{2} \mathrm{O}_{2}$ to protect plant cells from oxidative damage. The activities of two antioxidant enzymes that scavenge reactive oxygen species, superoxide dismutase (SOD) and catalase (CAT) in lettuce leaves in the presence of NO donor under PQ stress were higher than those under PQ stress alone. Application of 2-(4-carboxyphenyl)-4, 4, 5, 5-tetramethylimidazoline-1-oxyl-3- oxide (PTIO), a specific NO scavenger, to the lettuce leaves arrested SNP mediated protective effect on leaf growth, photosynthetic pigment and antioxidant systems. However, PTIO had little effect on lettuce leaves under PQ stress compared with that of PQ stress alone. The obtained data suggest that the damage caused by PQ stress is in part due to increased generation of active oxygen by maintaining increased antioxidant enzyme activities and SNP protects plants from oxidative stress. From these results it is suggested that NO might act as a signal in activating active oxygen scavenging system that protects plants from oxidative damage induced by PQ stress and thus confer PQ tolerance.
\end{abstract}

Key Words : Antioxidant enzymes, Lactuca sativa L., Nitric oxide, Oxidative stress, Paraquat

\section{Introduction}

A variety of toxic oxygen species such as superoxide, hydrogen peroxide, hydroxyl radicals and singlet oxygen are produced as a result of biochemical and physiological reactions within plant cells. Stress-induced accumulation of excessive quantities of reactive oxygen species (ROS) in plant cells is a typical phenomenon, because ROS-induced oxidative stress is an early damaging factor resulting

${ }^{*}$ Corresponding author : Jung-Hee Hong, Department of Biological Sciences, Pusan National University, Busan 609-735, Korea

Phone: +82-51-510-2263

E-mail: jhhong@pusan.ac.kr from stress treatment in plants (Shevyakova et al., 2009). ROS are toxic but also act as signaling molecules to mediate many key physiological processes. Plant cells have developed differential strategies to regulate their intracellular ROS concentrations by scavenging of ROS.

Toxic oxygen species are generated in the chloroplast during photosynthesis and the mechanisms have evolved by plants to scavenge these toxic oxygen species in the chloroplast (Shaaltiel and Gressel, 1986). The toxicity of active oxygen lies in the fact that it can react with numerous cell components thereby causing a cascade of oxidative reactions resulting in lipid peroxidation, protein degradation, inactivation of enzymes, DNA strand 
breaks, and pigment bleaching (Scandalios, 1993). The coordinated activities of antioxidant defense systems scavenging ROS protect plant cells from the deleterious effects of toxic oxygen species, which are produced at elevated rates when plants are exposed to environmental and xenobiotic stress conditions. Enzymatic antioxidant activity has been correlated with resistance to hyperoxia, photooxidative destruction by paraquat, salinity, ozone, chilling, drought and freezing (Kraus et al., 1995).

Herbicides cause loss of turgidity of affected plants, bleaching of leaves and death of plant. The deleterious effect of herbicides is believed to be strong oxidation of cell cell components such as lipid peroxidation in cellular membranes via free radical chain reactions (Schmidt and Kunert, 1986). The bipyridylium herbicide paraquat acts in chloroplasts in the light through the generation of superoxide by accepting electron from photosystem I (PSI) to become a reduced free radical, producing ROS. Because ROS damage membranes, inducing lipid peroxidation and result in chlorophyll bleaching in chloroplasts, detoxification of ROS plays an important role in tolerance of plants to paraquat stress (Kraus and Fletcher, 1994). Much evidences demonstrate that the resistance to paraquat was correlated with developmental stage and antioxidant enzyme activity, and the imposition of oxidative stress results in increases in substrates and gene products associated with scavenging cycle. Although these adaptive responses have the potential of conferring additional antioxidant resistance on the stressed plant cell, the chain of events linking the generation of ROS with antioxidant resistance responses is not yet understood.

Nitric oxide (NO) is a bioactive free radical implicated in a number of physiological functions including an important secondary messenger in animal cells and accumulating evidences suggest that it is important in plant cells as well (Leshem, 1996;
Wendehenne et al., 2001). NO has been proposed to be a functional metabolite as signaling molecule involved in growth, development and defense responses. NO was reported to play important roles in diverse physiological responses, such as growth, germination, senescence, apoptosis/programmed cell death, root organogenesis, pathogen response, phytoalexin production and regulation of stomatal closure (reviewed by Neill et al., 2003; Qiao and Fan, 2008). NO has also been involved in response to abiotic and biotic stresses, such as drought, salinity, heat, UV-B radiation and disease infection. Recently, it has been demonstrated that $\mathrm{NO}$ is involved in the protection system against oxidative stress induced by herbicide paraquat, a generator of superoxide radicals (Zhao et al., 2007). Based on these data, one can suggest that NO is involved in scavenging not only hydroxyl radical, but also superoxide radical, which trigger the chain of oxidative reactions in the cells.

Several reports demonstrate that NO is able to counteract the toxicity of paraquat and diquat, which are known to generate ROS in potato and rice leaves (Beligni and Lamattina, 1999; Hung et al., 2002), and block $\mathrm{H}_{2} \mathrm{O}_{2}$ production induced by cadmium in rice leaves (Hsu and Kao, 2004) or jasmonic acid in tomato leaves (Orozco-Cardenas and Ryan, 2002). It seems that NO has a important and innate role in combating stress effects. Thus, a possible participation of NO in antioxidant system against paraquatinduced stress is suggested. Nevertheless, further investigations would be needed to further elucidate the relationship between $\mathrm{NO}$ and oxidative damage under abiotic stresses, and the role of NO for inducing adaptive responses. The present study was conducted to determine whether NO confers tolerance to paraquat-mediated oxidative stress and to establish if tolerance is correlated with changes in the antioxidant system. 


\section{Materials and Methods}

\subsection{Plant material and growth conditions}

Seeds of lettuce (Lactuca sativa L.) were germinated in wet tissue paper and sown in pots containing a mixture of vermiculite and perlite $(1: 2, \mathrm{v} / \mathrm{v})$ in a growth chamber under a 15-h light / 9-h dark regime, $160 \mu \mathrm{mol}$ photons $\mathrm{m}^{-2} \mathrm{~s}^{-1}, 70 \pm 5 \% \mathrm{RH}$ and day/night temperatures of $20 / 15^{\circ} \mathrm{C}$. Plants were reared for 8 weeks under the same conditions and achieved full vegetative state.

The secondary leaves of the control and each experiments were detached from 8-week-old lettuce plants, and exposed to various treatments. Test solutions included paraquat, NO donor sodium nitroprusside (SNP) and NO scavenger 2-(4carboxyphenyl)-4, 4, 5-tetramethyl-imidazoline-1-oxy3-oxide (PTIO). For treatments, $200 \mu \mathrm{M} \mathrm{SNP}$ and PTIO were applied on the leaves at 6-h intervals for $24 \mathrm{~h}$, while only water was applied to the control plants.

For the pre-treatment, the detached leaves were floated on $20 \mathrm{ml}$ of SNP solution in Petri dishes for 3 $\mathrm{h}$ under a white light source, then placed in solutions containing paraquat, SNP or PTIO. For the paraquat application, a paraquat bioassay $(0-1.0 \mathrm{mM})$ was used to examine differential sensitivity to pigment bleaching and chlorophyll loss between control and paraquat-treated leaves. The concentration of $50 \mu \mathrm{M}$ paraquat showed moderate toxicity, thus was used to determine the effect of SNP on the PQ stressed plants.

For the induction of paraquat stress, $50 \mu \mathrm{M}$ paraquat was applied to the leaves that had been pretreated with or without SNP, either alone or in combination with $200 \mu \mathrm{M}$ SNP or PTIO under same growth conditions. After being exposed to various time periods, leaf samples were harvested daily, measured, frozen in liguid $\mathrm{N}_{2}$ and stored at $-70^{\circ} \mathrm{C}$ for further analysis.

\subsection{Growth measurements}

To evaluate leaf growth, leaf length, leaf width and leaf area were measured after $24 \mathrm{~h}$ of treatment. Leaf fresh weight was determined by using an electronic balance. Leaf dry weight was measured following over drying at $80^{\circ} \mathrm{C}$ for $72 \mathrm{~h}$ and reweighing. For the paraquat stress survival assays, leaves were monitored for bleaching and necrosis over $24 \mathrm{~h}$ and photographs taken at $0,6,12,24 \mathrm{~h}$ from one representative experiment were shown.

\subsection{Determination of photosynthetic pigments}

Detached leaves were frozen, then transferred to $\mathrm{N}, \mathrm{N}$-dimethylformamide and stored in the dark at $4^{\circ} \mathrm{C}$ until they were analyzed. The total chlorophyll (a and b) and carotenoid levels were measured spectrophotometrically at 3 different wavelengths : 663, 645 and $480 \mathrm{~nm}$. The concentration of chlorophyll and carotenoid contents were quantified according to the method of Inskeep and Bloom (1985).

2.4. Determination of flavonoids and anthocyanin contents

Flavonoids and anthocyanin were measured according to the method of Mirecki and Teramura (1984) with some modifications. Detached leaves (5 g) were homogenized using mortar and pestle at $4{ }^{\circ} \mathrm{C}$ in $5 \mathrm{ml}$ of extraction buffer (99: 1 methanol- $\mathrm{HCl}$, $\mathrm{v} / \mathrm{v})$. The homogenate was centrifuged at 2,000 $\mathrm{g}$ for $10 \mathrm{~min}$ at $4{ }^{\circ} \mathrm{C}$, and the supernatant was used for assays. Flavonoids and anthocyanin contents were estimated by measuring absorbances at 300 and 530 $\mathrm{nm}$, respectively.

\subsection{Chlorophyll fluorescence measurement}

Chlorophyll fluorescence induction parameters of leaves were measured at room temperature using a pulse-amplitude modulated fluorometer (PAM 2100, Walz, Germany) at the adaxial leaf surface of fresh plant material. Before each measurement, plants were dark-adapted for $10 \mathrm{~min}$, and then fluorescence data 
was collected during a 5-min continuous illumination using the LED of the PAM as the actinic light source $\left(63 \mu \mathrm{mol} \mathrm{m} \mathrm{m}^{-2}\right)$. Saturating pulses were given every $20 \mathrm{~s}$ and photochmical quantum yield of PS $\Pi$ was calculated (Schreiber et al., 1986). The maximal photochemical efficiency of PS $\amalg$ photochemistry was evaluated as $F v / F m=(F m-F o) / F m$ where $F o$ and $F m$ represent fluorescence levels under irradiation before and after a saturating pulse (3000 $\left.\mu \mathrm{mol} \mathrm{m} \mathrm{m}^{-2}\right)$, respectively.

\subsection{Determination of lipid peroxidation}

Lipid peroxidation was estimated by the level of malondialdehyde (MDA) production in thylakoid membrane preparation according to Zhao et al. (1994). Fresh leaves ( $0.5 \mathrm{~g})$ were homogenized in 10 $\mathrm{ml}$ of $5 \%$ trichloroacetic acid and the homogenate was then centrifuged at 4,000 $\mathrm{g}$ for $10 \mathrm{~min}$. The supernatant $(2 \mathrm{ml})$ was mixed with $2 \mathrm{ml}$ of $10 \%$ trichloroacetic acid containing $0.5 \%$ thiobarbituric acid, and then boiled at $100{ }^{\circ} \mathrm{C}$ for $20 \mathrm{~min}$. After centrifugation at $12,000 \mathrm{~g}$ for $10 \mathrm{~min}$, the absorbance of the supernatant was measured at 532 and $620 \mathrm{~nm}$ using a spectrophotometer. The amount of MDA was calculated using an extinction coefficient of 155 $\mathrm{mM}^{-1} \mathrm{~cm}^{-1}$.

\subsection{Determination of $\mathrm{H}_{2} \mathrm{O}_{2}$ content}

The contents of $\mathrm{H}_{2} \mathrm{O}_{2}$ were determined by peroxidecoupled assay according to Veljovic-Jovanovic et al. (2002). Detached leaves (1 g) were ground with a mortar and pestle in liquid nitrogen, and the powder was extracted in $2 \mathrm{ml}$ of $1 \mathrm{M} \mathrm{HClO}_{4}$ in the presence of insoluble polyvinylpyrrolidone $(5 \%, w / v)$. The homogenate was centrifuged at $12,000 \mathrm{~g}$ for $10 \mathrm{~min}$ at $4{ }^{\circ} \mathrm{C}$, and then the supernatant was neutralized to pH 7.5 with $5 \mathrm{M} \mathrm{K}_{2} \mathrm{CO}_{3}$ in the presence of $0.1 \mathrm{ml}$ of $0.3 \mathrm{M}$ phosphate buffer ( $\mathrm{pH}$ 5.6). The solution was centrifuged at $12,000 \mathrm{~g}$ at $4{ }^{\circ} \mathrm{C}$ for $1 \mathrm{~min}$, and the sample was incubated for $10 \mathrm{~min}$ with 1 unit of ascorbate oxidase to oxidize ascorbate prior to assay. The reaction mixture consisted of $0.1 \mathrm{M}$ phosphate buffer (pH 6.5), $3.3 \mathrm{mM}$ 3-(dimethylamino) benzoic acid, $0.07 \mathrm{mM}$ 3-methyl-2-benzothiazoline hydrazone, and 0.3 unit of peroxidase. This reaction was initiated by the addition of $0.2 \mathrm{ml}$ of sample. The absorbance change was monitored at $590 \mathrm{~nm}$ and compared with increases elicited by standard samples of hydrogen peroxide.

2.8. Determination of antioxidant enzyme activity

Activity of total superoxide dimutase (SOD) was determined by the method of Spychalla and Desborough (1990). The assay was performed at $25^{\circ} \mathrm{C}$ in a $3 \mathrm{ml}$ cuvette containing $50 \mathrm{mM} \mathrm{Na} \mathrm{CO}_{3} /$ $\mathrm{NaHCO}_{3}$ buffer (pH 10.2), 0.1 mM EDTA, 0.015 $\mathrm{mM}$ ferricytochrome $\mathrm{C}$ and $0.05 \mathrm{mM}$ xanthine. One unit of SOD is defined as the amount of enzyme that inhibited the rate of ferricytochrome $\mathrm{C}$ reduction by $50 \%$. Mn-SOD activity was determined as described above except that assay mixture contained $2 \mathrm{mM}$ $\mathrm{KCN}$ to inactive $\mathrm{Cu}, \mathrm{Zn}-\mathrm{SOD}$. $\mathrm{Cu}, \mathrm{Zn}-\mathrm{SOD}$ was calculated from the difference between total SOD and Mn-SOD activities.

Catalase (CAT) activity was assayed by monitoring the consumption of $\mathrm{H}_{2} \mathrm{O}_{2}$ (Durner and Klessing, 1996). The decrease in $\mathrm{H}_{2} \mathrm{O}_{2}$ was followed as the decline in absorbance at $240 \mathrm{~nm}$, and activity was calculated using the extinction coefficient $\left(39.4 \mathrm{mM}^{-1}\right.$ $\left.\mathrm{cm}^{1}\right)$. The reaction mixture contained $2 \mathrm{ml}$ of 100 $\mathrm{mM} \mathrm{NaH} \mathrm{PO}_{4} / \mathrm{Na}_{2} \mathrm{HPO}_{4}$ buffer ( $\mathrm{pH}$ 6.5), 50 pl of plant extract and the reaction was initiated by adding $10 \mu \mathrm{l}$ of $30 \%$ (w/v) $\mathrm{H}_{2} \mathrm{O}_{2}$.

\subsection{Statistical analysis}

Each experiment was analyzed at least three times, and a standard deviation (S.D.) was calculated. The data were expressed in mean \pm S.D. of three replicates. Statistical analysis was performed using the Student's t-test. 


\section{Results and Discussion}

3.1. Effect of NO on leaf growth under paraquat stress

To specify the role of NO under paraquat (PQ) stress, SNP as NO donor and PTIO as NO scavenger were applied to lettuce leaves. SNP-treated leaves were slightly shorter and wider than those of non-treated controls (Table 1). The most notable effect of PQ treatment is pigment bleaching. Severe leaf-damage symptoms including necrosis and wilting, were observed in lettuce leaves exposed to PQ alone (Fig. 1). The leaves affected by PQ toxicity showed chlorosis between veins and developed necrotic lesions with black spots. While those that had been pre-treated with SNP were less affected. SNP-treated leaves caused no severe leaf injuries, although symptoms of minor damage appeared. The SNP-induced morphological changes of lettuce leaves were consistent with those previously reported for other species (Leshem and Haramaty, 1996).

SNP-treated leaves maintained their initial fresh mass during $24 \mathrm{~h}$, while PQ application induced gradual decrease in leaf biomass by $24 \mathrm{~h}$ (Fig. 2A). The tendency of the rate of chlorophyll loss in the leaves was similar, indicating that SNP reduced the loss of chlorophyll (Fig. 2B). Fresh and dry-matter accumulations were remarkably suppressed by PQ alone or combined application (Fig. 3). The combination of PQ with SNP increased these parameters to a larger extent compared with PQ alone, demonstrating that NO increased the tolerance of plant to PQ. The application of SNP can induce rapid growth, exhibiting

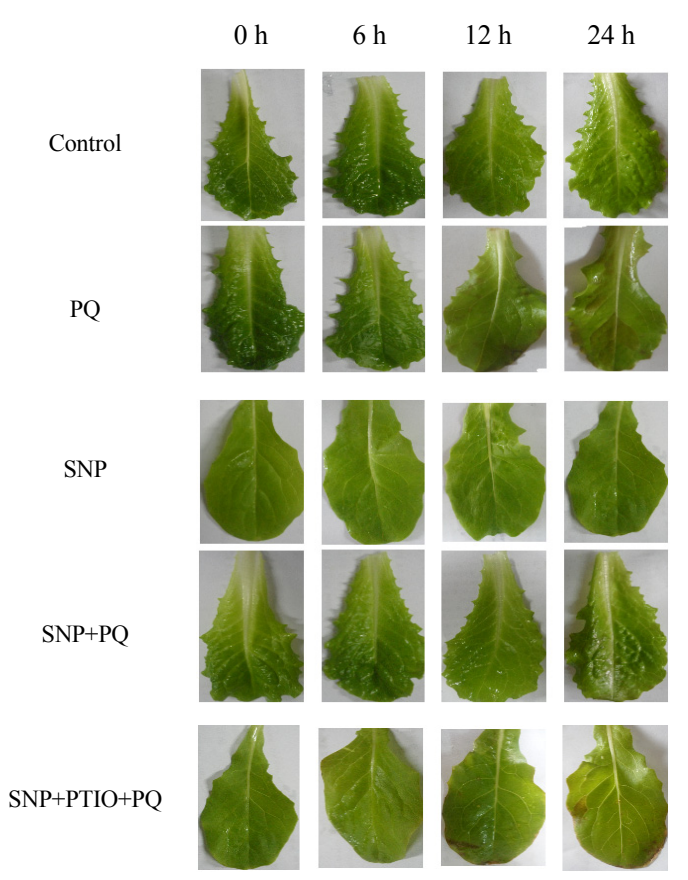

Fig. 1. Effect of SNP on the paraquat-induced lettuce leaf injury symptoms. Detached leaves were exposured to $50 \mu \mathrm{M}$ paraquat (PQ), $200 \mu \mathrm{M}$ SNP and PTIO alone or in combination for $24 \mathrm{~h}$.

thicker and greener than those of non-treated controls. The increased size accounts for the increased fresh mass of the leaves. However, high concentration of SNP ( $2 \mathrm{mM}$ ) inhibited wheat and pea growth, while low micromolor concentrations produced an increase in the rate of leaf expansion (Tu et al., 2003). This is possibly the result of diverse sensitivity of each organ to NO. From the above findings, it has been implicated that $\mathrm{NO}$ acts as a stimulator molecule in plant photomorphogenesis.

Table 1. Effect of SNP on the leaf growth of detached lettuce leaves exposed to paraquat

\begin{tabular}{lccc}
\hline Treatment & Leaf length $(\mathrm{cm})$ & Leaf width $(\mathrm{cm})$ & Leaf area $\left(\mathrm{cm}^{2} /\right.$ leaf $)$ \\
\hline \hline Control & $8.3 \pm 0.2$ & $3.7 \pm 0.1$ & $20.5 \pm 1.0$ \\
PQ & $8.0 \pm 0.4$ & $3.6 \pm 0.1$ & $19.7 \pm 1.0$ \\
SNP & $8.2 \pm 0.3$ & $3.8 \pm 0.2$ & $20.8 \pm 1.1$ \\
SNP + PQ & $8.1 \pm 0.3$ & $3.8 \pm 0.2$ & $20.5 \pm 1.0$ \\
SNP + PTI0 + PQ & $8.1 \pm 0.3$ & $3.7 \pm 0.1$ & $19.9 \pm 1.0$ \\
\hline
\end{tabular}




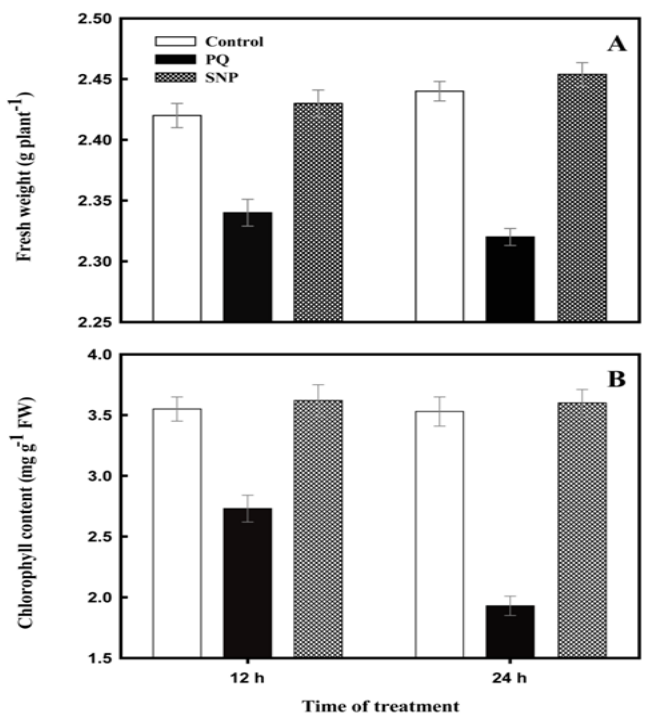

Fig. 2. Changes in the fresh weight(A) and chlorophyll content(B) in detached lettuce leaves treated with paraquat and SNP.

\subsection{Effect of NO on photosynthetic responses under paraquat stress}

Treatment with SNP prevented completely or alleviated the decrease of chlorophyll and carotenoid in lettuce leaves induced by PQ stress (Table 2). However, there was no pronounced difference in photosynthetic pigments between PQ alone and $\mathrm{PQ}+\mathrm{PTIO}$. From these findings, it is proven that PQ induced chlorophyll loss was reversed by NO donor and that NO has protective effects on the chloroplast membrane. It was shown that NO protects chloroplast levels in stressed leaves and NO participates in bringing about partial greening in etiolated seedlings

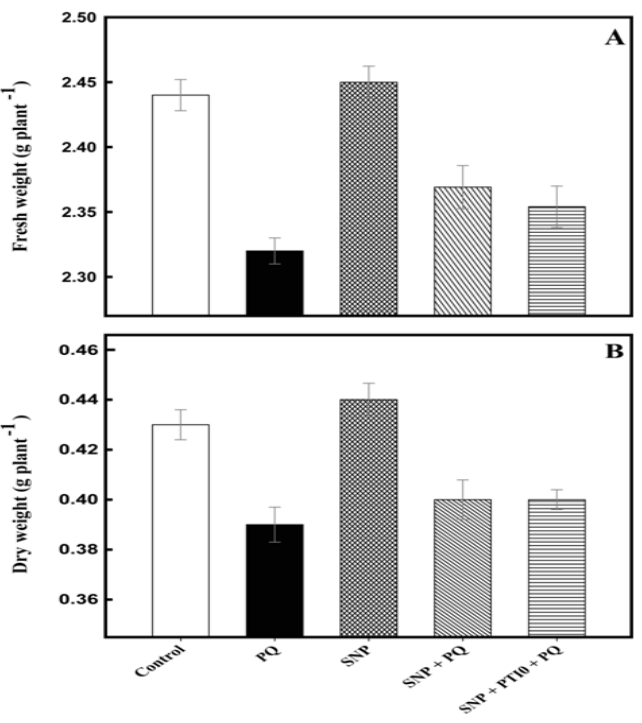

Fig. 3. Effect of SNP on the fresh weight(A) and dry weight(B) of detached lettuce leaves exposed to paraquat.

by the synergistic effect of NO and stress (Beligni and Lamattina, 2000).

The ratio of variable to maximal fluorescence $(\mathrm{Fv} / \mathrm{Fm})$ was determined as an indication of PS $\Pi$ efficiency (Bjorkman and Demming, 1987), and a decline in the ratio suggests photoinhibitory damage. Over the course of the experiment, the $F v / F m$ ratios declined by $59 \%$ in PQ-treated leaves, indicating that these leaves underwent some photoinhibition (Fig. 4). While SNP prevented this effect as $F v / F m$ ratios were maintained within the normal range. These findings demonstrate that the reduction in PS $\Pi$ activity was

Table 2. Effect of SNP on the chlorophyll and carotenoid contents in detached lettuce leaves exposed to paraquat

\begin{tabular}{lcc}
\hline Treatment & Chlorophyll content $\left(\mathrm{mg} \mathrm{g}^{-1} \mathrm{FW}\right)$ & Carotenoid content $\left(\mathrm{mg}^{-1} \mathrm{~g}^{-1} \mathrm{FW}\right)$ \\
\hline \hline Control & $3.527 \pm 0.141$ & $0.159 \pm 0.005$ \\
PQ & $1.930 \pm 0.077$ & $0.071 \pm 0.002$ \\
SNP & $3.620 \pm 0.110$ & $0.167 \pm 0.005$ \\
SNP + PQ & $3.355 \pm 0.134$ & $0.145 \pm 0.005$ \\
PT10 + PQ & $2.015 \pm 0.080$ & $0.080 \pm 0.003$ \\
SNP + PTI0 + PQ & $2.223 \pm 0.088$ & $0.092 \pm 0.003$ \\
\hline
\end{tabular}




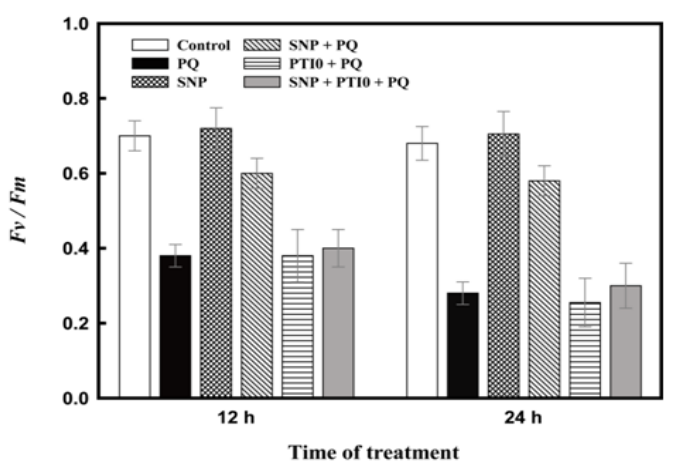

Fig. 4. Effect of SNP on the chlorophyll fluorescence in detached lettuce leaves exposed to paraquat.

largely prevented by SNP application and chloroplast membranes were protected to a greater extent from PQ stress. It was shown that SNP pretreatment reduced salt and heat stress induced damage in rice seedlings and prevented the impairment of PS $\amalg$ (Uchida et al., 2002).

\subsection{Effect of $\mathrm{NO}$ on antioxidant system under $\mathrm{PQ}$} stress

The contents of flavonoids and anthocyanin in PQ-treated leaves increased with increasing duration of incubation (Fig. 5). Accumulations in lettuce leaves $234 \%$ over the control for flavonoids and anthocyanin, were greater upon PQ exposure, being 189\% and respectively. In the presence of radiation, SNP intensified the product of flavonoids and anthocyanin. Comparatively, there was no difference between PTIO and PQ treatment.

Secondary metabolites, particularly phenylpropanoids, have long been recognized as serving key antioxidant role in response to wide range of environmental stimuli including UV-B and sunlight radiation. Tattini et al. (2004) suggested that flavonoids may serve antioxidant functions in response to drought and excess light stress. The strong association of anthocyanins with chlorophyllous cells has been shown to have a primary role in photosynthesis perhaps by protecting chloroplasts from photoinhibition



Fig. 5. Effect of SNP on the flavonoid(A) and anthocyanin(B) contents in detached lettuce leaves exposed to paraquat.

during periods of high photon flux.

The proposed roles for anthocyanin include protection from photoinhibition, defense against hervivores and free radical scavenging (Steyn et al., 2002). Anthocyanins in leaf tissues have a dual function as a absorber of harmful levels of radiation and osmotic adjuster. The rapid increase in these compounds was alleviated by SNP upon PQ exposure, which may perhaps be a defense response against PQ stress. The photoinduced accumulation of flavonoids and anthocyanin is preceded by the induction of several enzymes involved in phenyl- propanoid metabolism.

The content of malondialdehyde (MDA) is an indicator of lipid peroxidation and oxidative damage to the membrane due to free radicals. PQ exposure dramatically potentiated MDA after $24 \mathrm{~h}$ of incubation (Fig. 6). SNP treatment showed a distinct decrease when compared with PQ-induced MDA contents, demonstrating that NO confers a certain degree of protection to lettuce leaves from thylakoid membrane damage induced by lipid peroxidation. In contrast, 


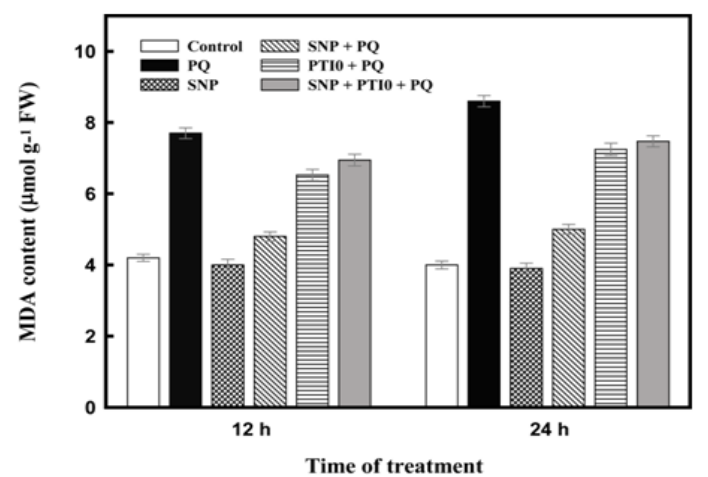

Fig. 6. Effect of SNP on the MDA content in detached lettuce leaves exposed to paraquat.

The combination of PQ with PTIO resulted in a remarkable increase in MDA contents, and MDA content in combination with SNP and PTIO was close to the level of PQ treatment alone. The effect of SNP on PQ stress and decrease in MDA contents could be recovered by PTIO. Judging from the lipid peroxidation activity, the exogenous SNP to lettuce leaves on the background of PQ treatments drastically decreased a degree of oxidative damage to the membrane under PQ Stress, but inconsiderably increased the chlorophyll in their chloroplast.

The oxidative stress generated under PQ stress acumulates hydrogen peroxide $\left(\mathrm{H}_{2} \mathrm{O}_{2}\right)$ as a main kind of ROS. Under PQ stress, $\mathrm{H}_{2} \mathrm{O}_{2}$ content in lettuce leaves significantly increased to $185 \%$ of the control, while it decreased to $41 \%$ in the presence of SNP (Fig. 7). Treatment with PTIO led to the reverse stimulating more $\mathrm{H}_{2} \mathrm{O}_{2}$ production. PTIO blocked the effect of SNP on the $\mathrm{H}_{2} \mathrm{O}_{2}$ accumulation in leaves, while PTIO itself had no influence on $\mathrm{H}_{2} \mathrm{O}_{2}$ content under PQ exposure. PTIO in combination with SNP induced an increase in $\mathrm{H}_{2} \mathrm{O}_{2}$ contents compared with those of PQ treatment alone.

Activities of SOD and catalase that remove $\mathrm{H}_{2} \mathrm{O}_{2}$ in plants were markedly increased in response to $24 \mathrm{~h}$ of exposure to PQ alone compared with untreated control leaves (Figs. 8 and 9). However, application of

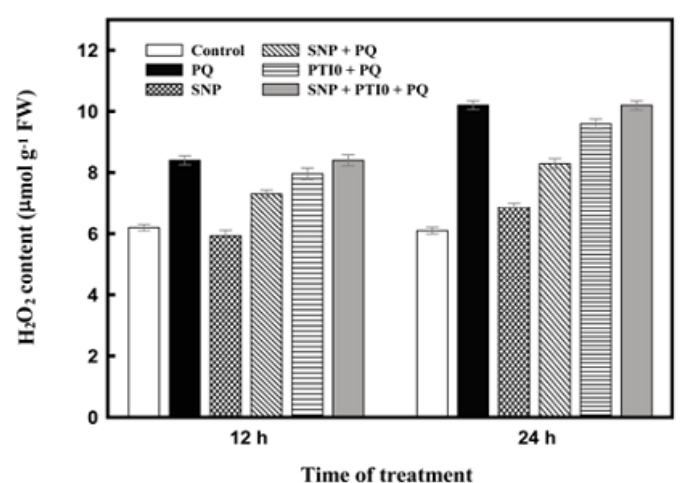

Fig. 7. Effect of SNP on the $\mathrm{H}_{2} \mathrm{O}_{2}$ content in detached lettuce leaves exposed to paraquat.

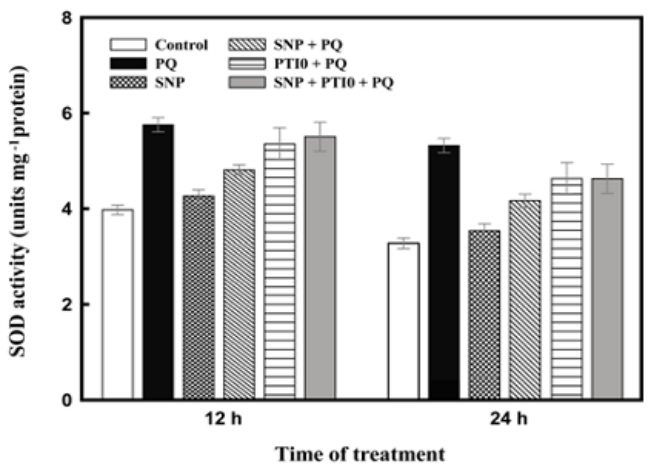

Fig. 8. Effect of SNP on the SOD activity in detached lettuce leaves exposed to paraquat.



Fig. 9. Effect of SNP on the catalase activity in detached lettuce leaves exposed to paraquat. 
SNP effectively retarded this rapid increase in enzyme activities that resulted from PQ stress. Their activities showed a similar pattern with increasing initially then decreasing over time under PQ exposure. For elucidation of SNP antioxidant role, it is important to note that exogenous SNP decreased PQ-induced SOD activities and consequently lowered the content of $\mathrm{H}_{2} \mathrm{O}_{2}$, the product of superoxide radical dismutase. Whereas catalase activity in leaves remained low. That is the reason why exogenous SNP could be a scavenger of superoxide radicals in the chloroplast. The protective effect of NO may also be related to its ability to react with some ROS making NO act as a chain breaker and show its proposed antioxidant properties. Our evidence supports the concept that $\mathrm{H}_{2} \mathrm{O}_{2}$ and $\mathrm{NO}$ can be important signal molecules for abiotic stress tolerance, although characterization of NO radicals as signal molecule in plants has been limited so far. The promotive effect of NO donor on the antioxidant enzymes was reversed in the presence of NO scavenger, PTIO. The combination of PQ with PTIO had lower CAT activities than PQ treatment alone.

Lipid peroxidation and the increase in the specific activities of antioxidant enzymes are consequence of ROS overproduction and NO acts as an ROS scavenger. NO alleviates the harmfulness of ROS, reacts with other target molecules, and regulates the expression of stress responsive genes under various stress conditions (Qiao and Fan, 2008). We also observed that SNP counteracted PQ-induced increase in the specific activities of antioxidant enzymes (SOD and CAT) and PTIO reversed the effect of SNP-increased specific activities. Clearly, the effect of NO donor SNP is attributable to NO released. The stimulating effect of NO donor were suppressed by the NO scavenger, indicating that NOS may play an important role in NO-mediated PQ-induced antioxidant enzyme activity (Tossi et al., 2009). PQ might first induce NO synthesis and the PQ-induced NO then stimulates the antioxidant enzymes.
The inducible effect of NO on the activity of antioxidant enzymes was observed in many other plant species under abiotic stresses such as drought, salinity, heat, herbicide and heavy metals (Zhao et al, 2007; Qiao and Fan, 2008). The present study also provides evidence that NO dependence on antioxidant activity serves as a signaling component in the induction of protective responses and is associated with PQ tolerance in lettuce plants. However, it was reported that CAT have been shown to be inhibited by NO in tobacco (Clark et al., 2000) and SNP treatment alone did not affect the specific activities of antioxidant enzymes in rice leaves (Hsu and Kao, 2004). Those lines of evidence implicate that the response to some stressors may be specific to certain species.

In the present paper, $\mathrm{NO}$ increased the tolerance to lettuce to PQ, suggesting that increased antioxidant defenses should confer cross-tolerance to several oxidative stresses. In conclusion, NO can induce the antioxidant system to prevent accumulation of ROS and protect plants from oxidative damage under PQ stress. Thus, it is suggested that NO is involved in the PQ signaling pathway and may serve as a secondary messenger to mediate adaptive response to PQ stress.

\section{Conclusions}

This study demonstrates that SNP protects lettuce leaves from injury due to paraquat stress. Application of paraquat induced photoinhibition, loss of fresh and dry mass and degradation of components of the photosynthetic machinery in lettuce leaves. This suggests that the chloroplast may be a primary site of damage caused by paraquat stress in illuminated plant tissues. Protection againt phytotoxic peroxidation is achieved by antioxidants such as NO donor, SNP. SNP, when applied exogenously during paraquat stress, is able to reverse induced loss of photosynthetic pigments and accumulation of antioxidant metabolites including anthocyanin and flavonoids. The protective 
effect of exogenous SNP was manifested in an increase in the chlorophyll content and a decline in lipid peroxidation and hydrogen peroxide. SNP counteracted paraquat-induced increase in the specific activities of antioxidant enzymes such as SOD and catalase. SNP stimulated an overall increase in antioxidant enzyme activities indicating that SNP elevated the plants to a high level of oxidative stress tolerance. However, the protective effect of SNP on the antioxidant system under paraquat stress was suppressed in the presence of NO scavenger, PTIO. These results demonstrate that NO donor (SNP) and scavenger (PTIO) alleviated and exaggerated the paraquat stress, respectively. Therefore, NO might mediate tolerance to elevated levels of paraquat toxicity and preclude oxidative damage.

\section{References}

Beligni, M. V., Lamattina, L., 2000, Nitric acid protects against cellular damage produced by methyl viologen herbicides in potato plants, Nitric Oxide Biol. Chem., 3, 199-208.

Beligni, M. V., Lamattina, L., 1999, Nitric oxide counteracts cytotoxic processes mediated by reactive oxygen species in plant tissues, Planta, 208, 337-344.

Bjorkman, O., Demmig, B., 1987, Photon yield of oxygen evolution and chlorophyll fluorescence characteristics at $77^{\circ} \mathrm{K}$ among vascular plants of diverse organ, Planta, 170, 489-504.

Clark, D., Dunar, J., Navarre, D. A., Klessig, D. F., 2000, Nitric oxide inhibition of tobacco catalase and ascorbate peroxidase, Mol. Plant-Microbe Interact., 14, 1380-1384.

Durner, J., Klessing, D. F., 1996, Salicylic acid is a modulator of tobacco and mammalian catalases, J. Biol. Chem., 271, 28492-28502.

Hsu, Y. T., Kao, C. H., 2004, Cadmium toxicity is reduced by nitric oxide in rice leaves, Plant Growth Regul., 42, 227-238.

Hung, K. T., Chang, C. J., Kao, C. H., 2002, Paraquat toxicity is reduced by nitric oxide in rice leaves, J. Plant Physiol., 159, 159-166.

Inskeep, W. P., Bloom, P. R., 1985, Extinction coefficients of chlorophyll a and b in N, N-dimethylformamide and $80 \%$ acetone, Plant Physiol., 77, 483-485.

Kraus, T. E., Fletcher, R. A., 1994, Paclobutrazol protects wheat seedlings from heat and paraquat injury: Is detoxification of active oxygen involved?, Plant Cell Physiol., 35, 45-52.

Kraus, T. E., McKersie, B. D., Fletcher, R. A., 1995, Paclobutrazol-induced tolerance of wheat leaves to paraquat may involve increased antioxidant enzyme activity, J. Plant Physiol., 145, 570-576.

Leshem, Y. Y., 1996, Nitric oxide in biological systems, Plant Growth Regul., 18, 155-159.

Leshem, Y. Y., Haramaty, E., 1996, The characterization and contrasting effects of the nitric oxide free radical in vegetative stress and senescence of Pisum sativum Linn. foliage, J. Plant Physiol., 148, 258-263.

Mirecki, R. M., Teramura, A. H., 1984, Effects of ultraviolet-B irradiance on soybean, Plant Physiol., 74, 475-480.

Neill, S. J., Desikan, R., Hancock, J. T., 2003, Nitric oxide signaling in plants, New Phytol., 159, 11-35.

Orozco-Cardenas, M., Ryan, C. A., 2002, Nitric oxide negatively modulate wound signaling in tomato plants, Plant Physiol., 130, 487-493.

Qiao, W., Fan, L. -M., 2008, Nitric oxide signaling in plant response to abiotic stresses, J. Int. Plant Biol., 50, 1238-1246.

Schmidt, A, Kunert, K. J., 1986, Lipid peroxidation in higher plants : The role of glutathione reductase, Plant Physiol., 82, 700-702.

Scandalios, J. G., 1993, Oxygen stress and superoxide dismutases, Plant Physiol., 101, 7-12.

Schreiber, U., Schliwa, U., Bilger, W., 1986, Continuous recording of photochemical and nonphotochemical chlorophyll fluorescence quencing with a new type of modulation fluorometer, Photosynth. Res., 10, 51-62.

Shaaltiel, Y., Gressel, J., 1986, Multienzyme oxygen radical detoxifying system correlated with paraquat resistance in Conyza bonariensis, Pestic. Biochem. Physiol., 26, 22-28.

Shevyakova, N. I., Bakulina, E. A., Kuznetsov, VI, V., 2009, Proline antioxidant role in the common ice plant subjected to salinity and paraquat treatment inducing oxidative stress, Russian Jour. Plant 
Physiol., 56, 663-669.

Spychalla, J. P., Desborough, S. L., 1990, Superoxide dismutase, catalase and alpha tocopherol content of stored potato tubers. Plant Physiol., 94 : 12141218.

Steyn, W. J., Wand, S. J. E., Holcroft, D. M., Jacob, G., 2002, Anthocyanins in vegetative tissues : A proposed unified function in photoprotection, New Phytol., 155, 349-361.

Tattini, M., Galardi, C., Pinelli, P., Massai, R., Remorini, D., Agati, G., 2004, Differential accumulation of flavonoids and hydroxycinnainates in leaves of Ligustrum vulgare under excess light and drought stress, New Phytol., 163, 547-561.

Tossi, V., Lamattina, L., Cassia, R., 2009, An increase in the concentration of abscisic acid is critical for nitric oxide-mediated plant adaptive responses to UV-B irradiation, New Phytol., 181, 871-879.

Tu, J., Shen, Y. B., Xu, L. L., 2003, Regulation of nitric oxide on the aging process of wheat leaves, Acta. Bot. Sin., 45, 1055-1062.
Uchida, A., Jagendorf, A. T., Hibino, T., Takabe, T., Takabe, T., 2002, Effects of hydrogen peroxide and nitric oxide on both salt and heat stress tolerance in rice, Plant Sci., 163, 515-523.

Veljovic-Jovanovic, S., Noctor, G., Foyer, C. H., 2002, Are leaf hydrogen peroxide concentration commonly overestimated? The potential influence of artefactual interference by tissue phenolics and ascorbate, Plant Physiol. Biochem., 40, 501-507.

Wendehenne, D., Pulgin, A., Klessing, D. F., Durner, J., 2001, Nitric acid : comparative synthesis and signaling in animal and plant cell, Trends Plant Sci., 6, 177-186.

Zhao, M. -G., Tian Q. -Y. Zhang, W. -H,, 2007, Nitric oxide synthase-dependent nitric oxide production is associated with salt tolerance in Arabidopsis, Plant Physiol., 144, 206-217.

Zhao, S. J., Xu, C. C., Zhou, Q., Meng, Q. W., 1994, Improvement of method for measurement of malondialdehyde in plant tissues, Plant Physiol., Comm., 30, 207-210. 